\title{
The role of transcatheter arterial embolization in patients with resectable hepatocellular carcinoma: a nation-wide, multicenter study
}

Huang Y-H, Chen C-H, Chang T-T, Chen S-C, Wang S-Y, Lee P-C, Lee H-S, Lin P-W, Huang G-T, Sheu J-C, Tsai H-M, Chau G-Y, Chiang J-H, Lui $\mathrm{W}-\mathrm{Y}$, Lee $\mathrm{S}-\mathrm{D}, \mathrm{Wu} \mathrm{J}-\mathrm{C}$. The role of transcatheter arterial embolization in patients with resectable hepatocellular carcinoma: a nation-wide, multicenter study.

Liver International 2004: 24: 419-424. (C) Blackwell Munksgaard 2004

Abstract: Purpose: The role of transcatheter arterial embolization (TAE) for patients with resectable hepatocellular carcinoma (HCC) is controversial. Analyzing a cohort of nation-wide data can delineate the beneficial effect of TAE for those patients. Methods: From 1991 to 1995, 818 patients who had potentially resectable HCC from four medical centers in Taiwan were enrolled. Among them, 599 underwent curative resection, 157 received TAE and 62 received supportive treatment alone. The survivals among the three groups were compared. Results: The 5-year survival rates for patients who underwent surgery, TAE and supportive treatment were $43.6 \%, 25.6 \%$ and $3.7 \%$, respectively. Surgery offered the best survival for those patients. TAE could also prolong survival as compared with supportive treatment $(P=0.0001)$. However, among patients who were in advanced tumor stage (Cancer and the Liver Italian Program (CLIP) score $\geq 2$ ), no statistical difference in survival was noted between patients who underwent TAE or supportive treatment. In multivariate analysis, single tumor, serum albumin $\geq 3.5 \mathrm{~g} / \mathrm{dl}$, tumor size less than $5 \mathrm{~cm}$, early-stage tumor (CLIP score $=0-1$ ) and aggressive treatment including surgery and TAE were independent factors associated with a better survival. Conclusions: Surgery is superior to TAE for patients with resectable HCC. In patients who refuse surgery, TAE can be considered for selected patients whose tumors are in early stage.

\section{Yi-Hsiang Huang ${ }^{1,2}$, Chien-Hung Chen ${ }^{3}$, Ting-Tsung Chang ${ }^{4}$, Shinn- Cherng Chen ${ }^{5}$, Shen-Yung Wang ${ }^{1}$, Pui-Ching Lee ${ }^{2}$, Hsuan-Shu Lee ${ }^{3}$, Pin-Wen Lin ${ }^{6}$, Guan-Tarn Huang ${ }^{3}$, Jin-Chuan Sheu ${ }^{3}$, Hong-Ming Tsai ${ }^{7}$, Gar-Yang Chau ${ }^{8}$, Jen-Huei Chiang ${ }^{9}$, Wing-Yiu Lui ${ }^{8}$, Shou-Dong Lee ${ }^{2}$ and Jaw-Ching $\mathbf{W u}^{1,2,10}$}

${ }^{1}$ Institute of Clinical Medicine, National YangMing University, Taipei, Taiwan, ${ }^{2}$ Division of Gastroenterology, Department of Medicine, Taipei Veterans General Hospital, Taipei, Taiwan, ${ }^{3}$ Department of Internal Medicine, National Taiwan University Hospital and National Taiwan University College of Medicine, Taipei, Taiwan, ${ }^{4}$ Division of Gastroenterology, National ChengKung University, Tainan, Taiwan, ${ }^{5}$ Hepatobiliary Division, Department of Internal Medicine, College of Medicine, Kaohsiung Medical University Hospital, Kaohsiung, Taiwan, ${ }^{6}$ Department of Clinical Medicine, National Cheng-Kung University, Tainan, Taiwan, ${ }^{7}$ Department of Radiology, College of Medicine, National Cheng-Kung University, Tainan, Taiwan, ${ }^{8}$ Department of Surgery, Taipei Veterans General Hospital, Taipei, Taiwan, ${ }^{9}$ Department of Radiology, Taipei Veterans General Hospital, Taipei, Taiwan, ${ }^{10}$ Department of Education and Research, Taipei Veterans General Hospital, Taipei, Taiwan.

Key words: CLIP score - hepatocellular carcinoma - surgery - transcatheter arterial chemoembolization

Prof. Jaw-Ching Wu, MD, PhD, National YangMing University, Division of Gastroenterology, Department of Medicine, Taipei Veterans General Hospital, 201 Shih-Pai Road, Sec. 2, Taipei 112, Taiwan.

Tel: 886228712121 ext: 3218

Fax: 886228745074

e-mail: jcwu@vghtpe.gov.tw

Received 2 October 2003

accepted 23 March 2004 
Hepatocellular carcinoma (HCC) is prevalent in Asia, Mediterranean countries and South Africa and ranks as the leading cause of cancer deaths for males in Taiwan (1). The incidence of HCC has been increasing in the United States as well as in the UK over the past two decades $(2,3)$. The prognosis of HCC is dismal. Surgical resection is the main choice of treatment for resectable HCC. However, the long-term survival of patients with HCC undergoing curative hepatic resection is still not satisfactory because of the high recurrence rate. Transcatheter arterial embolization (TAE), or, in association with intra-arterial chemotherapy (TACE) is mainly applied for patients who are unsuitable for surgical resection. The role of TAE/TACE in the treatment of $\mathrm{HCC}$ is still controversial. Among several randomized controlled trials comparing TAE/TACE with conservative treatment for unresectable $\mathrm{HCC}$, two show survival benefits favoring TAE/TACE, but the others demonstrate against the treatment (411). In regard to resectable $\mathrm{HCC}$, the effect of TAE/TACE is also debated (12-14). One study demonstrated that surgery could provide more favorable results in patients with early-stage HCC as compared with TAE (12). On the contrary, some reported a comparative 5-year survival for TAE or surgical resection in Child A and B or Okuda stage I patients (13-14). Noteworthily, those data were based on relative limited patient numbers or one hospital-based experience. Analyzing a cohort of nation-wide data can delineate the beneficial effect of TAE for patients with resectable HCC.

To evaluate the role of TAE/TACE on the survival rate for resectable $\mathrm{HCC}$ patients, we enrolled four medical centers under the support of the Department of Health (DOH), (Taiwan) to conduct this study. From 1991 to 1995, all HCC patients who underwent surgical resection or had the potential for surgical resection in four medical centers in Taiwan were retrospectively reviewed and studied.

\section{Patients and methods}

Patients and data collection

Four medical centers including National Taiwan University Hospital, Taipei; Taipei Veterans General Hospital, Taipei; National Cheng-Kung University Hospital, Tainan and Kaohsiung Medical University Hospital, Kaohsiung were enrolled in this study. These four medical centers encompass half of the main medical university hospital in Taiwan and are located two in northern Taiwan and two in southern Taiwan. The medical records of all HCC patients who visited these four medical centers from January 1991 to December 1995 were retrospectively reviewed.

There were totally 599 patients who underwent curative resection of $\mathrm{HCC}$ with complete medical records and pathology proven at the four medical centers. The criteria for HCC patients to undergo tumor resection included: (A) Child's grade of liver function was $\mathrm{A}$ or $\mathrm{B}$ with an indocyanine green (ICG) 15-min retention rate less than $30 \%$; (B) patients with tumors involving no more than three Healey's segments, without portal vein main trunk involvement or distant metastasis; (C) absence of other major diseases that may complicate the surgery for HCC (15). In the same period, 219 who fulfilled the above criteria but did not receive surgery were included. The diagnosis of HCC for the 219 patients was confirmed by biopsy or by $\alpha$-fetoprotein (AFP) assay $\geq 400 \mathrm{ng} / \mathrm{ml}$ or by focal lesions from at least two image examinations (sonography, computerized scan and celiac angiography) associated with progressive course of tumors (increasing size and number). All patients did not have severe liver disease, which was defined by the presence of one of the following: gastrointestinal hemorrhage in the past month, clinical ascites, edema or encephalopathy. Of the 219 patients, 157 patients received TACE (20 patients in Cheng-Kung University Hospital received TAE without intraarterial chemotherapy) because of their unwillingness to receive surgery and 62 patients refused aggressive treatment and received supportive treatment alone. The TAE was carried out through selective hepatic arterial catheterization; whenever possible, the arteries supplying the tumor(s) were catheterized superselectively followed by infusion of 5-10 $\mathrm{ml}$ of lipiodol (Laboratoire Guerbet, Paris, France). The feeding arteries were then embolized with $2-3 \mathrm{~mm}$ strips of gelfoam (Upjohn Co., Kalamazoo, MI ). TACE was carried out with the adjuvant of adriamycin in the remaining three medical centers.

The following variables were used for analysis: age and sex of patients, tumor number, tumor size, vascular invasion (including portal vein thrombosis), AFP levels (ng/ml), Child-Pugh class and serum alanine aminotransferase (ALT), albumin, total bilirubin levels and Cancer and the Liver Italian Program (CLIP) scores at diagnosis $(16,17)$.

Serum hepatitis B surface antigen (HBsAg) was tested using a radioimmunoassay kit (Abbott Laboratories, North Chicago, IL). Serum AFP was also measured using a radioimmunoassay kit (Serono Diagnostic SA, Coinsin/VD, Switzerland). Serum albumin, ALT and bilirubin, were measured by a systemic multiautoanalyzer (Tech- 
TAE for resectable $\mathrm{HCC}$

nicon SMAC, Technicon Instruments Corp., Tarrytown, NY).

\section{Identification of mortality}

We adopted the national citizen identification number of the studied patients to match with the mortality databank established by the Statistics Office, Department of Health, Taiwan. The mortality databank was based on the data in the Certificate of Death, which contained time, place and cause of death and details of the person who issued the document. The underlying cause of death was classified according to the International Classification of Disease.

\section{Statistical analysis}

The Wilcoxon rank sum test and the Student's $t$-test or $\chi^{2}$ test were used where appropriate for the comparison of variables among groups. When comparing the variables among the three groups, a one-way ANOVA was used. If the $P$-value was $<0.05$, a multiple comparison test using the Bonferroni test was used to compare the two groups. Cumulative survival rate was obtained by using the Kaplan-Meier method (18). Survival curves were statistically compared using the log-rank test. Variables that achieved statistical significance $(P<0.05)$ or were close to significance $(P<0.1)$ in the univariate analysis were subsequently included in a multivariate analysis using a stepwise forward Cox regression procedure (19). For all tests, a $P$-value $<0.05$ was considered significant.

\section{Results}

Clinical profile of patients with resectable $\mathrm{HCC}$

As shown in Table 1, the demographic data of patients with resectable HCC who received surgery, TAE/TACE or supportive treatment were comparable in sex, AFP levels, serum albumin, total bilirubin, ALT levels, the proportion of vascular invasion and the distribution of single or multinodular tumors, tumor size, Child-Pugh classification and CLIP scores. The mean age for patients receiving surgery was significantly younger than those who received TAE/TACE or supportive treatment. But the mean age between patients who received TAE/TACE and supportive treatment was not statistically different. The mean tumor size was larger in patients who received surgery than in who received TAE/ TACE $(P=0.013)$. There was no statistical difference in the mean tumor size between surgery

Table 1. Demographic data of the potentially resectable patients who underwent surgery, TAE or supportive treatment

\begin{tabular}{|c|c|c|c|c|}
\hline & Surgery $(n=599)$ & TAE/TACE $(n=157)$ & Supportive Tx $(n=62)$ & $P$-value \\
\hline Age (mean $\pm \mathrm{SD}$ ) & $60.0 \pm 12.2^{*}$ & $64.7 \pm 10.9$ & $62.5 \pm 12.0$ & $<0.001$ \\
\hline $\operatorname{Sex}(M: F)$ & $498: 101$ & $133: 24$ & $50: 12$ & 0.76 \\
\hline \multirow[t]{2}{*}{ Single tumor vs. multinodular tumors (\%) } & $448(75)$ & $104(66.2)$ & $45(72.6)$ & \multirow[t]{2}{*}{0.1} \\
\hline & $151(25)$ & $53(33.8)$ & $17(27.4)$ & \\
\hline Tumor size (mean $\pm \mathrm{SD}$ ) & $5.7 \pm 3.9 \dagger$ & $4.7 \pm 3.2$ & $5.5 \pm 3.2$ & 0.006 \\
\hline$<3 \mathrm{~cm}$ & $161(26.9)$ & $56(35.7)$ & $13(21)$ & \multirow[t]{4}{*}{0.288} \\
\hline$\geq 3$ and $<5 \mathrm{~cm}$ & $161(26.9)$ & $39(24.8)$ & $21(33.9)$ & \\
\hline$\geq 5$ and $<10 \mathrm{~cm}$ & $186(31.1)$ & $42(26.8)$ & $18(29)$ & \\
\hline$\geq 10 \mathrm{~cm}$ & $91(11.1)$ & $20(12.7)$ & $10(16.1)$ & \\
\hline $\operatorname{AFP}(\mathrm{ng} / \mathrm{ml})$ (median) & $52.4(1.34-244030)$ & $149(1.47-469540)$ & $144.5(2-198216)$ & 0.6 \\
\hline$<400$ & $405(67.6)$ & $96(61.1)$ & $36(58.1)$ & 0.134 \\
\hline$\geq 400$ & $194(32.4)$ & $61(38.9)$ & $26(41.9)$ & \\
\hline Albumin $(\mathrm{g} / \mathrm{dl})$ & $3.9 \pm 0.5$ & $3.8 \pm 0.5$ & $3.9 \pm 0.5$ & 0.092 \\
\hline Total bilrubin (mg/dl) & $1.1 \pm 0.7$ & $1.0 \pm 0.4$ & $1.0 \pm 0.4$ & 0.125 \\
\hline Alanine transaminase $(\mathrm{U} / \mathrm{l})$ & $61 \pm 57.5$ & $67.8 \pm 56.3$ & $74.8 \pm 59.8$ & 0.111 \\
\hline \multicolumn{5}{|l|}{ Child-pugh (\%) } \\
\hline A & $541(90.3)$ & $135(86)$ & $56(90.3)$ & \multirow[t]{2}{*}{0.28} \\
\hline B & $58(9.7)$ & $22(14)$ & $6(9.7)$ & \\
\hline Vascular invasion (\%) & $67(11.2)$ & $17(10.8)$ & $12(19.4)$ & 0.15 \\
\hline \multicolumn{5}{|l|}{ No. of CLIP score (\%) } \\
\hline 0 & $231(38.6)$ & $42(26.8)$ & $24(38.7)$ & \multirow[t]{5}{*}{0.201} \\
\hline 1 & $202(33.7)$ & $60(38.2)$ & $17(27.4)$ & \\
\hline 2 & $105(17.5)$ & $34(21.7)$ & $14(22.6)$ & \\
\hline 3 & $48(8)$ & $16(10.2)$ & $4(6.5)$ & \\
\hline 4 & $13(2.2)$ & $5(3.2)$ & $3(4.8)$ & \\
\hline HBsAg-positive (\%) & $395 / 568(69.5)$ & $93 / 142(65.5)$ & $37 / 61(60.7)$ & \multirow[t]{2}{*}{0.28} \\
\hline Anti-HCV-positive (\%) & $127 / 427(29.7)$ & $44 / 100(44)$ & $16 / 39(41)$ & \\
\hline
\end{tabular}

*Surgery vs. TAE/TACE $P<0.001$; surgery vs. supportive, $P<0.001$. $†$ Surgery vs. TAE/TACE, $P=0.013$; TAE/TACE vs. supportive, $P=0.458$. SD, Standard deviation; TAE, transcatheter arterial embolization; TACE, transcatheter arterial chemotherapy; AFP, $\alpha$-fetoprotein; CLIP, Cancer and the Liver Italian Program; HBsAg, Hepatitis B Surface antigen; HCV, Hepatitis C Virus. 
Huang et al.

and supportive treatment groups or between TAE/TACE and supportive treatment groups $(P=1.0$ and 0.458 , respectively). More than $60 \%$ of the patients were infected by hepatitis B virus (HBV). The anti-hepatitis $\mathrm{C}$ virus (HCV) data were not available for some patients because the assay was not widely used to survey HCC in early 1990s. One-third (33\%) of the 566 tested patients were infected by HCV.

Survival analysis by treatment modality (Fig. 1)

Among the 818 patients who had resectable HCC, surgery offered the best survival (Fig. 1). The median survival for patients who underwent surgery was 47.5 months $(95 \%$ confidence interval $(C I)=40.4-54.6$ months), which was significantly longer than 26.5 months for TAE/TACE (95\% CI $=19.5-33.5$ months, $P<0.0001)$ and 17.4 months (95\% CI $=11.9-22.9$ months) for supportive treatment. TAE/TACE could also significantly prolong the survival as compared with supportive treatment $(P=0.0001)$. The 5year survival rates for patients who underwent surgery, TAE/TACE and supportive treatment were $43.6 \%, 25.6 \%$ and $3.7 \%$, respectively.

Survival analysis of treatment modality stratified by CLIP score (Fig. 2)

As the previous report suggested TAE had a survival benefit for patients with early-stage HCC (13). We stratified patients into early (CLIP 0-1) and advanced stage (CLIP $\geq 2$ ) HCC for analysis. Among the 576 patients whose tumor stage was CLIP 0 or 1 , surgery and TAE/TACE could prolong the survival as compared with supportive treatment (Fig. 2A). However, for the 242
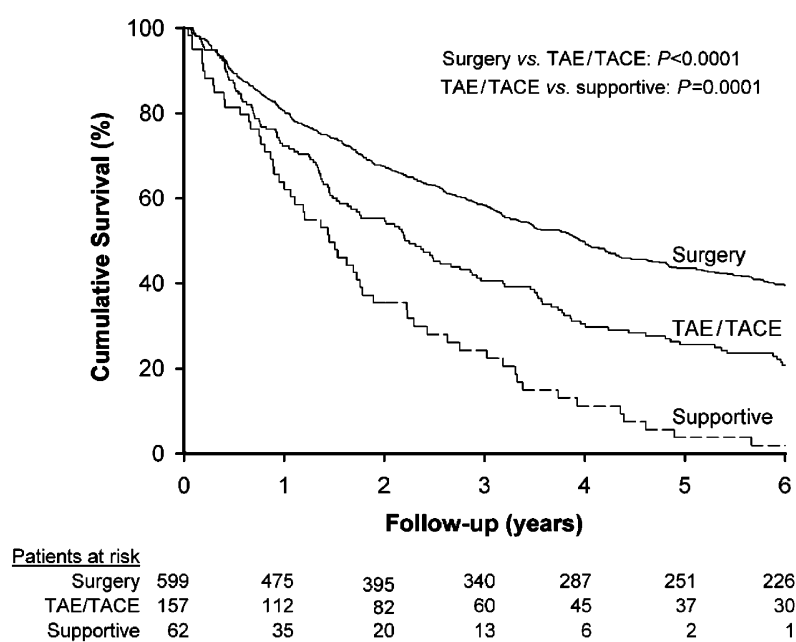

Fig. 1. Cumulative survival of patients, with hepatocellular carcinoma, who received surgery, transcatheter arterial embolization/chemotherapy (TAE/TACE) or supportive treatment.

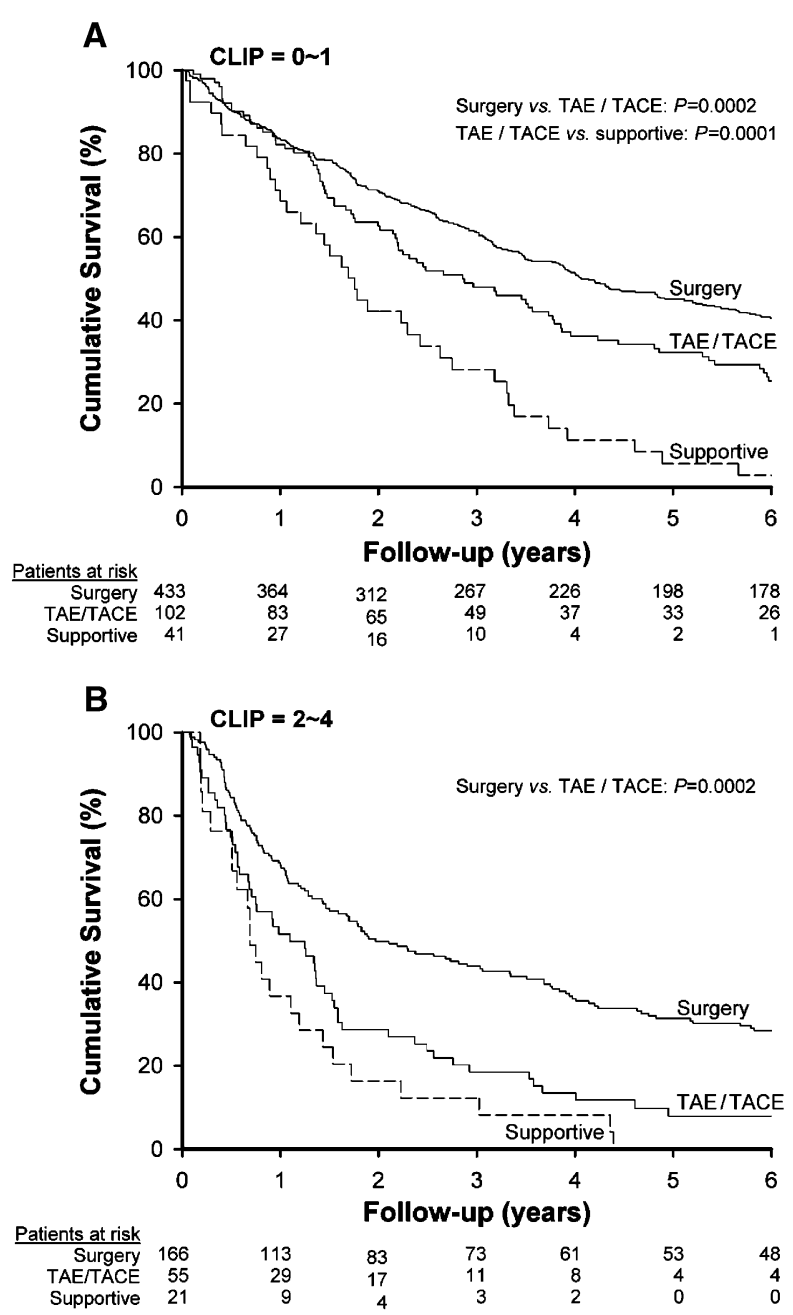

Fig. 2. Cumulative survivals of patients who received surgery, transcatheter arterial embolization/chemotherapy (TAE/TACE) or supportive treatment in Cancer and Liver Italian Program (CLIP) score 0 and 1 (A) or in CLIP score 2-4 (B).

patients whose HCC stage was CLIP 2-4, the median survivals were 27.5 months for surgery, 15.1 months for TAE/TACE and 10.7 months for supportive treatment. There was no statistical difference in survivals between patients who underwent TAE/TACE or supportive treatment at these stages $(P=0.286)$ (Fig. $2 \mathrm{~B})$.

\section{Univariate and multivariate analyses of factors associated with survival}

Several factors including age, sex, serum albumin, bilirubin, ALT levels, tumor number, vascular invasion, tumor size, AFP levels, treatment modality, Child-Pugh classifications and CLIP scores were analyzed for their association with survivals for 818 resectable HCC patients. Among them, age $<60$ years old, serum albumin $\geq 3.5 \mathrm{~g} / \mathrm{dl}$, $\mathrm{AFP}<400 \mathrm{ng} / \mathrm{ml}$, tumor size $<5 \mathrm{~cm}$, single tumor, Child A stage, CLIP score $\leq 1$ and treatment modalities were factors associated with 
TAE for resectable $\mathrm{HCC}$

longer survivals in univariate analysis (Table 2). In multivariate analysis, serum albumin $\geq 3.5 \mathrm{~g} /$ $\mathrm{dl}$, tumor size less than $5 \mathrm{~cm}$, single tumor, CLIP score $\leq 1$ and aggressive treatment (surgery or TAE/TACE) were still significantly associated with a better survival rate (Table 3 ).

Table 2. Univariate analysis of factors associated with survivals in 818 HCC patients

\begin{tabular}{|c|c|c|c|}
\hline Variables & $\begin{array}{l}\text { Patient } \\
\text { numbers }\end{array}$ & $\begin{array}{l}\text { Median survivals } \\
\text { (months) }(95 \% \mathrm{Cl})\end{array}$ & $P$-value \\
\hline \multicolumn{4}{|l|}{ Age (years) } \\
\hline$<60$ & 358 & $46(37.3-54.7)$ & \multirow[t]{2}{*}{0.011} \\
\hline$\geq 60$ & 460 & $37.1(32-42.2)$ & \\
\hline \multicolumn{4}{|l|}{ Sex } \\
\hline Male & 681 & $38(32.2-43.8)$ & \multirow[t]{2}{*}{0.596} \\
\hline Female & 137 & $42.7(35.1-50.3)$ & \\
\hline \multicolumn{4}{|l|}{ Albumin $(\mathrm{g} / \mathrm{dl})$} \\
\hline$\geq 3.5$ & 572 & $41.3(36.4-46.2)$ & \multirow[t]{2}{*}{0.0002} \\
\hline$<3.5$ & 146 & $25.2(17.7-32.7)$ & \\
\hline \multicolumn{4}{|l|}{ Bilirubin (mg/dl) } \\
\hline$<1.4$ & 651 & $33.2(23.7-42.7)$ & \multirow[t]{2}{*}{0.181} \\
\hline$\geq 1.4$ & 167 & $40.6(35.7-45.5)$ & \\
\hline \multicolumn{4}{|l|}{$\operatorname{ALT}^{-}(\mathrm{U} / \mathrm{I})$} \\
\hline$<120$ & 725 & $37.1(32.8-41.4)$ & \multirow[t]{2}{*}{0.301} \\
\hline$\geq 120$ & 93 & $51.7(43.4-60)$ & \\
\hline \multicolumn{4}{|l|}{ Child } \\
\hline A & 732 & $40.6(35.8-45.4)$ & \multirow[t]{2}{*}{0.022} \\
\hline $\mathrm{B}$ & 86 & $27.6(14.4-40.8)$ & \\
\hline \multirow{2}{*}{$\begin{array}{l}\text { Single vs. multinodular } \\
\text { tumors }\end{array}$} & 597 & $44.8(39-50.6)$ & \multirow[t]{2}{*}{$<0.0001$} \\
\hline & 221 & $24(17.2-30.8)$ & \\
\hline \multicolumn{4}{|l|}{ Tumor size $(\mathrm{cm})$} \\
\hline$<5$ & 451 & $48.4(42.3-54.5)$ & \multirow[t]{2}{*}{$<0.0001$} \\
\hline$\geq 5$ & 367 & $23.6(18.7-28.5)$ & \\
\hline \multicolumn{4}{|l|}{ Vascular invasion } \\
\hline Yes & 97 & $21.4(9.5-33.3)$ & \multirow[t]{2}{*}{0.0001} \\
\hline No & 721 & $41.8(36.8-46.8)$ & \\
\hline \multicolumn{4}{|l|}{$\operatorname{AFP}(\mathrm{ng} / \mathrm{ml})$} \\
\hline$<400$ & 537 & $45.7(41-50.4)$ & \multirow[t]{2}{*}{0.0164} \\
\hline$\geq 400$ & 281 & $24.5(17.9-31.1)$ & \\
\hline \multicolumn{4}{|l|}{ CLIP score } \\
\hline $0-1$ & 576 & $46.4(40.7-52.1)$ & \multirow[t]{2}{*}{$<0.0001$} \\
\hline $2-4$ & 242 & $19.1(13.6-24.6)$ & \\
\hline \multicolumn{4}{|l|}{ Treatment } \\
\hline Surgery & 599 & $47.5(40.4-54.6)$ & \multirow[t]{3}{*}{$<0.0001$} \\
\hline TAE/TACE & 157 & $26.5(19.5-33.5)$ & \\
\hline Supportive & 62 & $17.4(11.9-22.9)$ & \\
\hline
\end{tabular}

AFP, $\alpha$-fetoprotein; ALT, alanine aminotransferase; TAE, transcatheter arterial embolization; TACE, transcatheter arterial chemotherapy; CLIP, Cancer and the Liver Italian Program; HCC, hepatocellular carcinoma.

Table 3. Multivariate analysis of factors associated with longer survivals in 818 HCC patients

\begin{tabular}{lllr}
\hline & $\begin{array}{l}\text { Regression } \\
\text { Variable }\end{array}$ & & \\
\hline Surgery vs. supportive & 1.003 & $2.725(2.033-3.650)$ & $<0.001$ \\
TAE/TACE vs. supportive & 0.502 & $1.653(1.199-2.278)$ & 0.002 \\
Albumin $\geq 3.5 \mathrm{~g} / \mathrm{dl}$ & 0.322 & $1.381(1.127-1.692)$ & 0.002 \\
Tumor size $<5 \mathrm{~cm}$ & 0.375 & $1.455(1.222-1.732)$ & $<0.001$ \\
Single tumor & 0.349 & $1.418(1.174-1.714)$ & $<0.001$ \\
CLIP score $\leq 1$ vs. $\geq 2$ & 0.249 & $1.283(1.055-1.559)$ & 0.012 \\
\hline
\end{tabular}

TAE, transcatheter arterial embolization; TACE, transcatheter arterial chemotherapy; CLIP, Cancer and the Liver Italian Program.

\section{Discussion}

It is widely accepted that TAE is a palliative treatment for unresectable HCC. A recent metaanalysis study supports the beneficial effect of TAE for patients with unresectable HCC (20). Surgery is theoretically the first choice for patients with resectable HCC. However, some patients are unwilling to undergo surgery because of fear of operation or family's opposition. Whether TAE can serve as an alternative treatment for those patients deserves further survey. Only few authors compared the survival of TAE with that of surgery (12-15). In this study, we included a large scale of patients for analysis. In this studied population, most of the demographic data were nearly comparable among the three groups of patients, except for age and tumor size. According to the medical records and our experience in clinical practice, older age is the main reason for patients to refuse surgery. This was why patients who underwent surgery in the present study were younger than those who received TAE/TACE or supportive treatment. But age was not the factor associated with survival in the final multivariate analysis. Although tumor size was one of the factors associated with survival, the distribution of tumor size had no statistical difference among the three groups. Accordingly, the current data support that surgery is better than TAE to offer a longer survival for patients with resectable HCC.

TAE also has a survival benefit for patients with resectable $\mathrm{HCC}$, which is confirmed by multivariate analysis. Note worthily, the beneficial role of TAE is more significant for patients with early-stage HCC. Previous studies had demonstrated that the potential benefit of TACE might counteract by its harmful effect on liver function $(6,9)$. It is interesting to find that TAE has only marginal effect for patients whose tumors are in CLIP 2-4. The compositions of the CLIP score include the Child-Pugh stage, tumor morphology, AFP and portal vein thrombosis $(16,17)$. For patients with advanced CLIP score, the preserved liver function is poorer and the tumor is more extensive. Liver damage caused by TAE/TACE in patients with advanced CLIP score may be more severe and could offset its anti-tumor effect. Such patients must be carefully selected if TAE is considered.

Several factors including tumor number, tumor size and serum albumin level have been reported to be factors associated with survivals in HCC patients $(15,21,22)$. Compatible with those studies, serum albumin $\geq 3.5 \mathrm{~g} / \mathrm{dl}$, tumor size less than $5 \mathrm{~cm}$, single tumor were also the factors associated with longer survivals. Tumor stage is 
also one of the factors associated with survivals of patients with HCC. Recently, the CLIP staging system has proven to be better than the Okuda staging system to predict survival (23). In the present study, we also found that early CLIP stage is an independent prognostic factor for patients with resectable HCC.

TAE usually applies a chemotherapeutic agent, including adriamycin, cisplatin or mitomycin $\mathrm{C}$, during the procedure. Among the four medical centers in Taiwan, adriamycin is the most commonly used agent in TACE. It is widely accepted that TACE is a more rational therapy than TAE alone (24). However, definite evidence from randomized studies is still lacking. In this study, TAE and TACE were included together to analyze the survivals, because the survivals of patients who received TAE alone at Cheng-Kung University Hospital was comparable with those who received TACE in the remaining three medical centers (data not shown). Other locoregional therapies, such as percutaneous ethanol injection, microwave coagulation or radiofrequency ablation therapy were not included as these procedures were not widely used in Taiwan during that period.

In conclusion, surgery is superior to TAE for patients with resectable HCC. In patients who refuse surgery, TAE has a survival benefit for selected patients whose HCC are in early stage.

\section{Acknowledgement}

This study was supported by grants from the Department of Health (DOH91-HP-1009), Taiwan.

\section{References}

1. Department of Health, ROC. Cancer Registry Annual Report. 1998.

2. El-Serag H B, Mason A C. Rising incidence of hepatocellular carcinoma in the United States. N Engl J Med 1999; 340: $745-50$.

3. TAylor-Robinson S D, Foster G R, Arora S, et al. Increase in primary liver cancer in UK, 1979-94. Lancet 1997; 350: 1142-3.

4. Lin D Y, Liaw Y F, LeE T Y, et al. Hepatic arterial embolization in patients with unresectable hepatocellular carcinoma - a randomized controlled trial. Gastroenterology 1988; 94: 453-6.

5. Pelletier G, Roche A, Ink O, et al. A ramdomized trial of hepatic arterial chemoembolization in patients with unresectable hepatocellular carcinoma. J Hepatol 1990; 11: $181-4$.

6. GETCH. A comparison of lipiodol chemoembolization and conservative treatment for unresectable hepatocellular carcinoma. N Engl J Med 1995; 332: 1256-61.

7. Madden M V, Krige J E, Bailey S, et al. Randomized trial of targeted chemotherapy with lipiodol and 5-epidoxorubicin compared with symptomatic treatment for hepatoma. Gut 1993; 34: 1598-1600.
8. Bruix J, Llovet J M, Castells A, et al. Transarterial embolization versus symptomatic treatment in patients with advanced hepatocellular carcinoma: results of a randomized, controlled trial in a single institution. Hepatology 1998; 27: 1578-83.

9. Pelletier G, Ducreux M, Gay F, et al. Treatment of unresectable hepatocellular carcinoma with lipiodol chemoembolization: a multicenter randomized trial. J Hepatol 1998; 29: 129-34.

10. Llovet J M, Real M I, Montana X, et al. Arterial embolisation or chemoembolisation versus symptomatic treatment in patients with unresectable hepatocellular carcinoma: a randomised controlled trial. Lancet 2002; 359: 1734-9.

11. Lo C M, Ngan H, Tso W K, et al. Randomized controlled trial of transarterial lipiodol chemoembolization for unresectable hepatocellular carcinoma. Hepatology 2002; 35: 1164-71.

12. Kanematsu T, Matsumata T, Shirabe K, et al. A comparative study of hepatic resection and transcatheter arterial embolization for the treatment of primary hepatocellular carcinoma. Cancer 1993; 71: 2181-6.

13. Bronowicki J P, Boudjema K, Chone L, et al. Comparison of resection, liver transplantation and transcatheter oily chemoembolization in the treatment of hepatocellular carcinoma. J Hepatol 1996; 24: 293-300.

14. Yoshimi F, Nagao T, Inoue S, et al. Comparison of hepatectomy and transcatheter arterial chemoembolization for the treatment of hepatocellular carcinoma: necessity for prospective randomized trial. Hepatology 1992; 16: 702-6.

15. Huang Y H, Wu J C, Chau G Y, et al. Supportive treatment, resection and transcatheter arterial chemoembolization in resectable hepatocellular carcinoma: an analysis of survival in 419 patients. Eur J Gastroenterol Hepatol 1999; 11: 315-21.

16. The Cancer of the Liver Italian Program (CLIP) investigators. A new prognostic system for hepatocellular carcinoma: a retrospective study of 435 patients. Hepatology 1998; 28: 751-5.

17. The Cancer of the Liver Italian Program (CLIP) investigators. Prospective validation of the CLIP score: a new prognostic system for patients with cirrhosis and hepatocellular carcinoma. Hepatology 2000; 31: 840-5.

18. Kaplan G L, Meier P. Nonparametric estimation for incomplete observations. J Am Stat Assoc 1958; 53: 453-81.

19. Cox D. Regression models and life tables. J R Stat Soc (B) 1972; 34: 187-220.

20. Llovet J M, Bruix J. Barcelona-Clinic Liver Cancer Group. Systemic review of randomized trials for unresectable hepatocellular carcinoma: chemoembolization improves survival. Hepatology 2003; 37: 429-42.

21. Yamanaka N, Oкамото E, Toyosaka A, et al. Prognostic factors after hepatectomy for hepatocellular carcinoma. A univariate and multivariate analysis. Cancer 1990; 65: 1104-10.

22. Poon R T P, NGan H, Lo C M, et al. Transarterial chemoembolization for inoperable hepatocellular carcinoma and postresection intrahepatic recurrence. J Surg Oncol 2000; 73: 109-14.

23. Levy I, Sherman M. The Liver Cancer Study Group of the University of Toronto. Staging of hepatocellular carcinoma: assessment of the CLIP, Okuda, and Child-Pugh staging systems in a cohort of 257 patients in Toronto. Gut 2002; 50: 881-5.

24. Poon R T P, Fan S T, Tsang F H F, et al. Locoregional therapies for hepatocellular carcinoma: a critical review from the surgeon's perspective. Ann Surg 2002; 235: 466-86. 